\title{
A Case of Medullary Infarction Presented Initial Symptoms Similar to Meniere's Disease
}

\author{
Jang Hee Park, Dong Hyun Kim, Jae Moon Sung, and Chang Woo Kim \\ Department of Otorhinolaryngology-Head and Neck Surgery, Hallym University College of Medicine, Seoul, Korea
}

$\begin{array}{ll}\text { Received } & \text { June 7, 2017 } \\ \text { Revised } & \text { June 27, 2017 } \\ \text { Accepted } & \text { June 28, 2017 }\end{array}$

Address for correspondence

Chang Woo Kim, MD

Department of Otorhinolaryngology-

Head and Neck Surgery,

Hallym University

College of Medicine,

150 Seongan-ro, Gangdong-gu,

Seoul 05355, Korea

Tel +82-2-2224-2279

Fax +82-2-482-2279

E-mail kcw5088@dreamwiz.com
Fluctuating hearing loss and vertigo are the typical presentations of Meniere's disease. How ever, it is unusual that fluctuating hearing loss and vertigo are caused by vertebral artery occlusion or cerebral infarction. Here, we described the case of a 54-year-old male patient with hypertension and diabetes mellitus who presented with fluctuating hearing loss in his left ear and severe whirling-type dizziness without associated neurological signs or symptoms. Temporal magnetic resonance imaging (MRI) was normal. He was diagnosed with a possible Meniere's disease and started conservative treatment. Eight years later, the patient developed dysarthria and left-side weakness. Brain MRI revealed right anterior medullary infarction, and cerebral angiography showed occlusion of the right vertebral artery. In this case, we attempted to review the initial imaging study and reported the characteristics of the case.

J Audiol Otol 2018;22(1):48-52

KEY WORDS: Sudden hearing loss · Meniere's disease · Magnetic resonance imaging · Vertebral artery.

\section{Introduction}

Meniere's disease is a clinical disease characterized by recurrent paroxysmal dizziness, loss of hearing, earfullness, and tinnitus. The diagnosis can be made when a paroxysmal or rotatory dizziness lasting more than 20 min occurs more than two times, the hearing loss is confirmed by the hearing test, and the tinnitus or earfullness is accompanied by the ipsilateral side. However, long-term follow-up or treatment is required even if no typical symptoms are observed [1]. The first attack of Meniere's disease may occur in the form of sudden hearing loss. Sudden hearing loss is defined as an acute sensorineural hearing loss of more than $30 \mathrm{~dB}$ over three or more consecutive frequencies in a pure-tone audiometry test [2]. Sudden hearing loss has several causes, such as viral infections, vascular disorders, cochlear membrane rupture, autoimmune disease, and auditory neoplasm, which are rarely caused by early cerebral infarction without neurologic abnormalities $[3,4]$. Therefore, efforts to find the cause of sudden hearing loss are

This is an Open Access article distributed under the terms of the Creative Commons Attribution Non-Commercial License (http://creativecommons.org/licenses/by-nc/4.0/) which permits unrestricted non-commercial use, distribution, and reproduction in any medium, provided the original work is properly cited. necessary. If dizziness or auditory variability occurs, longterm follow-up should be performed because of the possibility of Meniere's disease. At this time, the imaging test for differential diagnosis of the cerebral lesion is mainly performed by magnetic resonance imaging (MRI) on the brain parenchyma. However, evaluation of the cerebral blood vessels is often neglectful.

We reported a case of anterior medullary infarction, which presented with recurrent unilateral hearing loss and dizziness and was initially diagnosed as possible Meniere's disease [5]. We reviewed the imaging studies and reported the characteristics of the cases.

\section{Case Report}

Written informed consent was obtained from the patient for publication of this report.

A 54-year-old man visited our clinic with a sudden onset of dizziness and left-sided hearing loss a day before. The patient was diagnosed with hypertension and diabetes for six months and was on medication. The dizziness was rotatory and persisted for several minutes, but the symptoms of nausea and vomiting were not severe. The left-side ear was accom- 
panied by earfullness and tinnitus similar to a "wing" sound, and no sensory abnormality of the face, headache, diplopia, swallowing disorder, consciousness disorder, or movement disorder was observed. The otoscopic examination was normal on both sides of the ear. In the pure-tone hearing test, the left side showed sensorineural hearing loss localized to the low- frequency region (Fig. 1A) and the right side was normal. No prolongation of the latency period was found in the auditory brainstem response test, and no nystagmus was observed in the spontaneous nystagmus test, gaze nystagmus test, and positioning nystagmus test. Although the patient was recommended hospitalization for the sudden onset of hearing loss
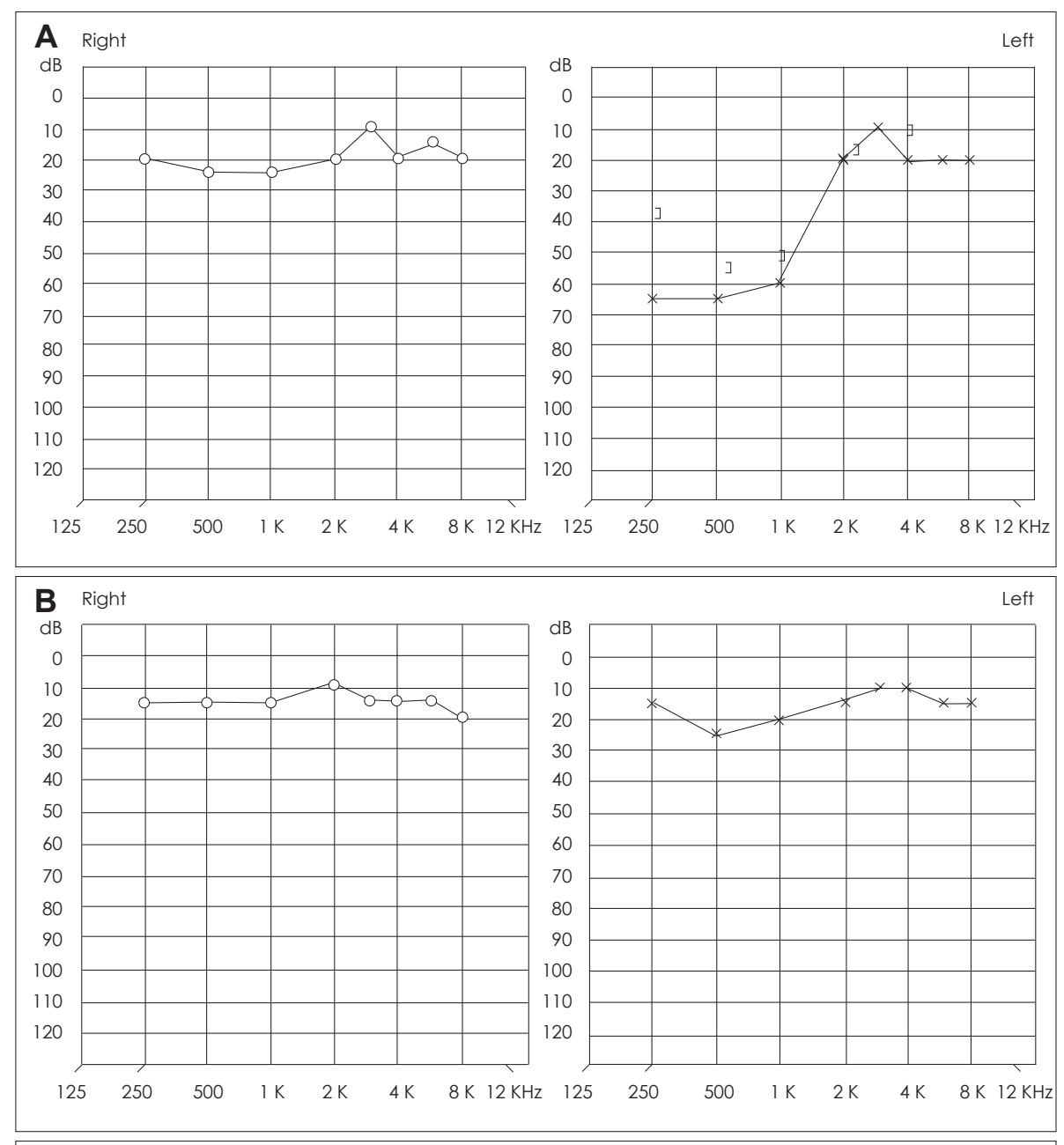

Fig. 1. Images of the pure-tone audiogram. A: The initial examination shows low frequency hearing loss in the left ear. B: Five days after the initial examination, the audiogram shows improvement of left-side hearing to normal. C: Twelve days after the initial examination, the audiogram shows low frequency hearing loss in the left ear. dB: decibel, $\mathrm{Hz}$ : hertz.

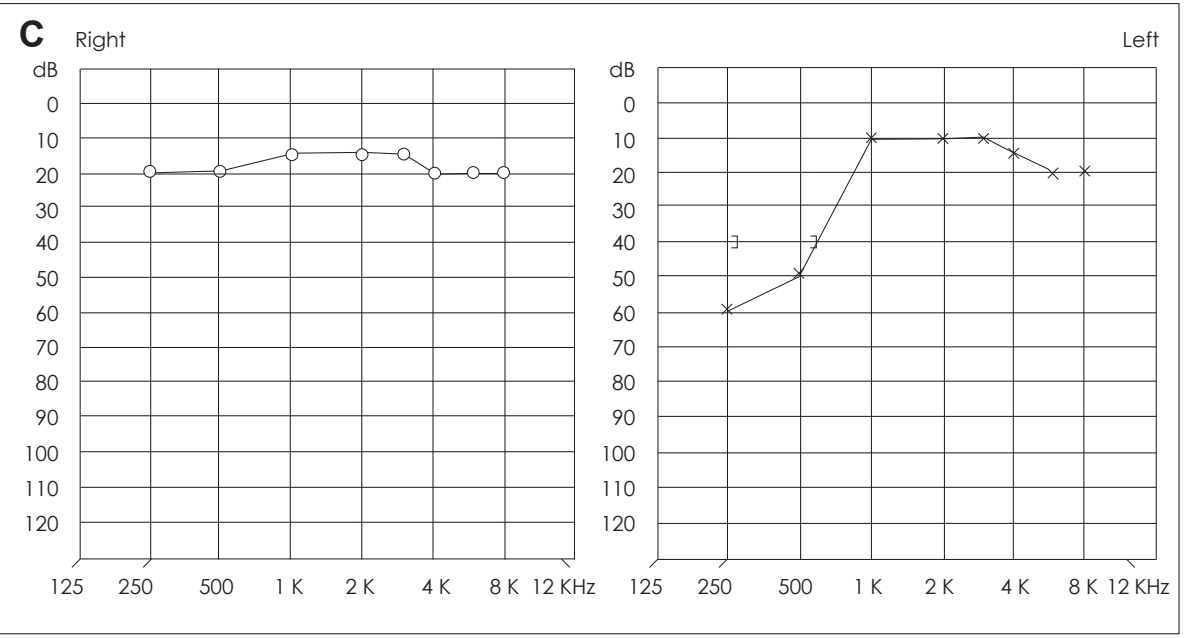


with dizziness, he decided to proceed with steroid therapy on the outpatient basis for personal reasons. Five days later, hearing was restored to normal range (Fig. 1B) and dizziness improved, but the left low-frequency hearing was reduced again after one week (Fig. 1C). Temporal bone magnetic resonance imaging revealed no evidence of abnormal lesions in bilateral internal auditory canal and brain parenchyma. The patient was diagnosed with a possible Meniere's disease and started conservative treatment at the outpatient clinic. After one year of follow-up, left-sided hearing loss and dizziness did not worsen, but the follow-up was lost afterward. Seven years later, he visited the emergency room with dizziness, vomiting, and headache. The symptoms subsided after intravenous hydration and medication. Three days later, a non-rotatory dizziness occurred and left tinnitus became severe. According to the pure-tone audiometry, left-side hearing remained the same, spontaneous nystagmus was not observed, and nystagmus occured after head shaking. Romberg's test, gait test, finger-tonose test, and heel-to-shin test were all normal. The patient complained of a headache in the occipital area, and dysarthria was observed. MRI was performed after admission to the neurology department to determine the central cause. Right anterior medullary infarction was observed in the diffusion weighted brain MRI (Fig. 2A). Additional CT angiography showed right vertebral artery occlusion (Fig. 2B), and antiplatelet therapy was started. No abnormal eye movements, ptosis, and facial paralysis were observed. In the evening of on the day of hospitalization, ataxic hemiparesis of the left upper and lower limbs occurred. After two weeks of inpatient treatment, the patient was discharged from the hospital with some restora-
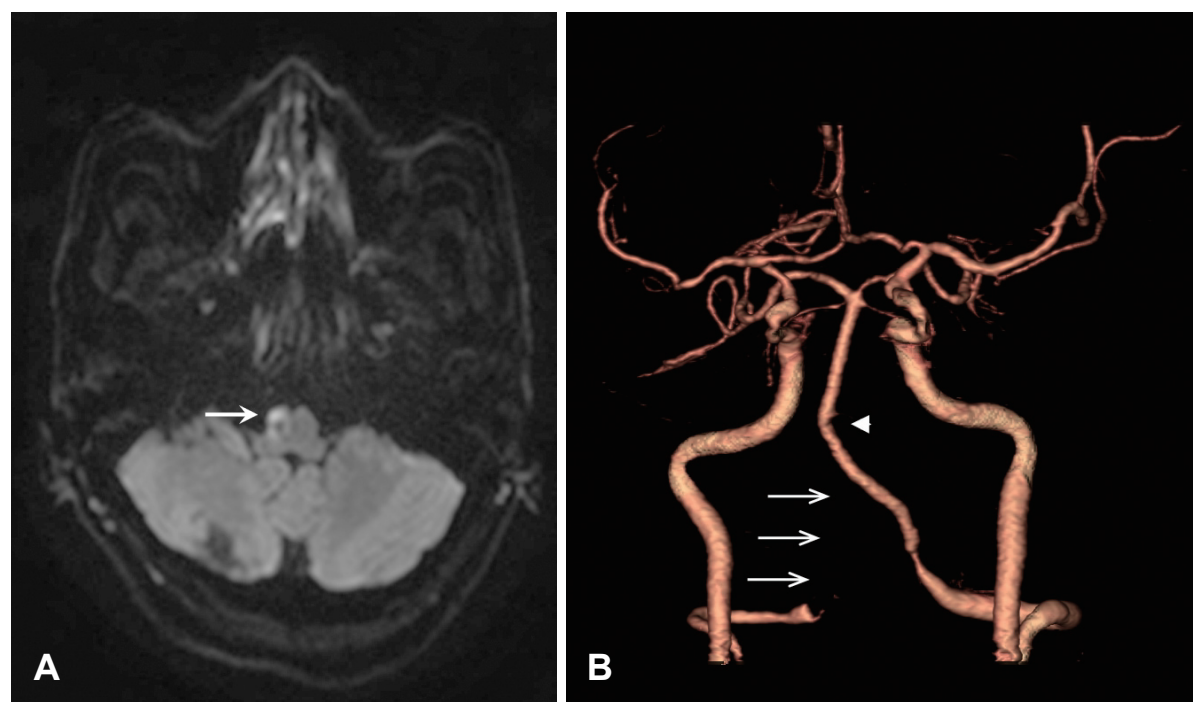

Fig. 2. Imaging features of the patient. A: Diffusion-weighted MRI shows high signal intensity on the right anterior medulla (arrow). B: Computed tomographic angiography shows near total occlusion of the right vertebral artery (arrows). Note that the basilar artery is curved toward the right side (arrowhead). MRI: magnetic resonance imaging.
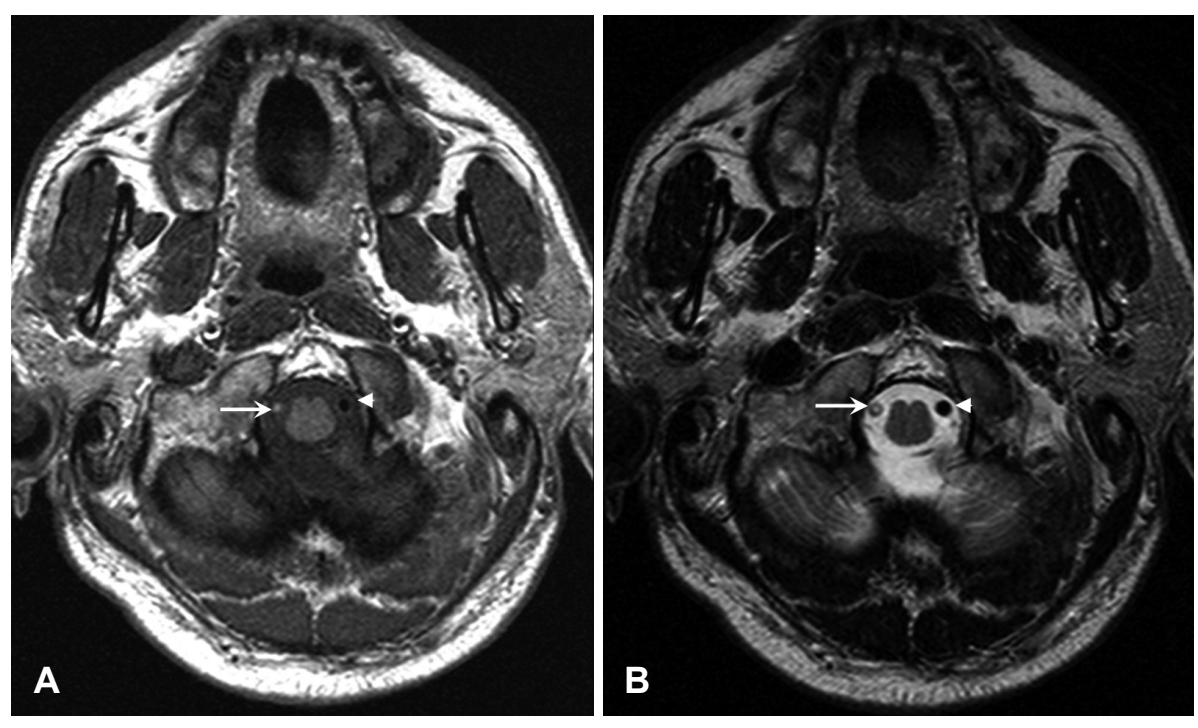

Fig. 3. Magnetic resonance images performed eight years ago. A: T1weighted MRI shows iso-signal or slightly hyperintense signal of the right vertebral artery (arrow) and hypo-signal intensity of the left vertebral artery (arrowhead). B: T2-weighted MRI shows disappearance of flow void signal of the right vertebral artery (arrow) and flow void signal of the normal left vertebral artery (arrowhead). MRI: magnetic resonance imaging. 
tion of dysarthria and ataxic hemiparesis of the left limb.

\section{Discussion}

In case of sudden hearing loss or Meniere's disease, the neurological signs and symptoms are important for the determination of central causes. If a central cause is suspected, MRI is performed for confirmation. In most cases, an imaging test for brain parenchyma is performed. However, even if the initial MRI scan is normal, a transient ischemic attack or a cerebral infarction may develop after the passage of time [6-8]. The elapsed time between the initial normal MRI scan and the onset of a stroke is between one day and two weeks, with most occurrences happening within five days [7]. In this case, initial MRI is normal because of infarction of the inner ear artery alone but progresses to cerebral infarction over time.

In the present case, right anterior medullary infarction, which was considered to be due to right vertebral artery occlusion, occurred. MRI of the temporal bone was performed at the time of diagnosis, assessed to be normal, and diagnosed as possible Meniere's disease with left-sided acute hearing loss and fluctuating dizziness. However, when the MRI image was reviewed after the infarction, abnormal findings were observed (Fig. 3). The right vertebral artery showed moderate or high signal intensity in both T1- and T2-weighted images showed moderate or high signal intensity. In the MRI, blood vessels showed no signal intensity (flow void) due to rapid blood flow in the blood vessels. When blood vessels are narrowed or obstructed, signal intensity changes may occur. In general, T1-weighted images are not used for the diagnosis of vascular occlusion because of increased signal intensity in the blood or cerebrospinal fluid flowing by flow-related enhancement [9]. In T2-weighted images, in the case of a coronal plane or a sagittal plane, the signal may be increased by a partial volume effect, but in the axial plane perpendicular to the blood vessel, it can be used as a reliable finding for the status of the internal carotid artery of skull base in the intracranial vertebral artery and the cerebral artery [10]. However, as its sensitivity is low, it is not used as a primary test for the diagnosis of cerebrovascular occlusion. An increase in signal intensity may be helpful when suspecting cerebrovascular anomalies. In this case, MRI examination of the brain parenchyma performed eight years ago was normal. However, the T2-weighted image showed suspicion of right vertebral artery occlusion, which seemed to be consistent with the cerebral angiographic findings eight years later. However, whether the left and fluctuating acute hearing loss was associated with right vertebral artery occlusion remained unclear. Note that the cerebral angiogram showed that the basilar artery was curved to the right side (Fig. 2B). In general, the vertebral artery has different sizes of the internal diameter between the two sides, and the resultant asymmetric flow pattern contributes to the development of the basilar artery curvature in the opposite direction of the dominant vertebral artery [11]. As a result, stretching and thinning of the anterior inferior cerebellar artery (AICA) in the opposite direction of the basilar artery curvature may be observed concurrently with shear stress-induced atherosclerosis in the inner wall of the basilar artery [11]. This morphological change may contribute to the development of sudden hearing loss on the same side as the vertebral artery dominancy [11]. In this case, we conjecture that the right vertebral artery occlusion caused an increase in blood flow in the left vertebral artery, thus resulting in a flexion of the basilar artery to the right, deformation of the left AICA, and ischemic injury to the left labyrinthine artery.

In this case, fluctuating sudden hearing loss and dizziness occurred at the initial stage, but brain MRI was assessed to be normal and conservative treatment was performed. Eight years later, right anterior medullary infarction and right vertebral artery occlusion were found. After reviewing the initial MRI scan, we suspected right vertebral artery abnormality on the T2-weighted images. If we had confirmed it through by cerebral angiography at that time, the occurrence of infarction might have been prevented. Therefore, in the case of acute inner ear dysfunction, examining the cerebral blood vessels and the MRI for brain parenchyma is necessary to discriminate central cause, and a detailed examination of T2-weighted images is helpful.

\section{Conflicts of interest}

The authors have no financial conflicts of interest.

\section{REFERENCES}

1) Ahsan SF, Standring R, Wang Y. Systematic review and meta-analysis of Meniett therapy for Meniere's disease. Laryngoscope 2015;125: 203-8.

2) Wilson WR. Why treat sudden hearing loss. Am J Otol 1984;5:481-3.

3) Son EJ, Bang JH, Kang JG. Anterior inferior cerebellar artery infarction presenting with sudden hearing loss and vertigo. Laryngoscope 2007;117:556-8.

4) Park SY, Shin JW, Cho YJ, Hong KS. Two cases of sudden hearing loss with vertigo as an isolated symptom of anterior inferior cerebellar artery infarction. J Korean Bal Soc 2008;7:85-8.

5) Committee on Hearing and Equilibrium. Committee on Hearing and Equilibrium guidelines for the diagnosis and evaluation of therapy in Menière's disease. American Academy of Otolaryngology-Head and Neck Foundation, Inc. Otolaryngol Head Neck Surg 1995;113:181-5.

6) Kim GW, Park JH, Kim DH, Kim CW. A case of acute inner ear dysfunction as a prodrome of transient ischemic attack caused by vertebral artery stenosis. Korean J Otolaryngol-Head Neck Surg 2016;59: 58-63.

7) Kim HS, Oh JI, Park MI, Kim CW. A case of anterior inferior cerebellar artery infarction initially presented a sudden sensorineural 
hearing loss with a normal diffusion-weighted brain MRI. Res Vestib Sci 2009;8:147-51.

8) Kim JS, Cho KH, Lee H. Isolated labyrinthine infarction as a harbinger of anterior inferior cerebellar artery territory infarction with normal diffusion-weighted brain MRI. J Neurol Sci 2009;278:82-4.

9) Caruso RD, Postel GC, McDonald CS, Sherry RG. High signal on T1-weighted MR images of the head: a pictorial essay. Clin Imaging 2001;25:312-9.
10) Li Q, Tian CL, Yang YW, Lou X, Yu SY. Conventional T2-weighted imaging to detect high-grade stenosis and occlusion of internal carotid artery, vertebral artery, and basilar artery. J Stroke Cerebrovasc Dis 2015;24:1591-6.

11) Kim C, Sohn JH, Jang MU, Hong SK, Lee JS, Kim HJ, et al. Ischemia as a potential etiologic factor in idiopathic unilateral sudden sensorineural hearing loss: analysis of posterior circulation arteries. Hear Res 2016;331:144-51. 